Keiko Wakui · Atsushi Toyoda · Takeo Kubota

Eiko Hidaka • Masayo Ishikawa • Tsutomu Katsuyama

Yoshiyuki Sakaki • Masahira Hattori • Yoshimitsu Fukushima

\title{
Familial 14-Mb deletion at 21q11.2-q21.3 and variable phenotypic expression
}

Received: March 1, 2002 / Accepted: June 14, 2002

\begin{abstract}
We report a familial case with a proximal interstitial deletion of chromosome $21 \mathrm{q}[\operatorname{del}(21 \mathrm{q})]$. Although the mother in the family was phenotypically normal, her first child was affected with both sensorineural hearing loss and moderate mental retardation, and the second affected child had mild mental retardation but not sensorineural hearing loss. We determined breakpoints of the $\operatorname{del}(21 \mathrm{q})$ in the mother and her two affected children by fluorescence in situ hybridization analysis using 45 DNA clones and the molecular analysis using 21 DNA markers. The proximal breakpoint of the del(21q) was located at a region between $0.33 \mathrm{Mb}$ and $0.46 \mathrm{Mb}$ distal to the centromere, and the distal breakpoint was at a region between $14.6 \mathrm{Mb}$ and $14.9 \mathrm{Mb}$. The finding indicates that the three individuals had an approximate 14-Mb deletion within 21q11.2-q21.3. Molecular analysis showed that both affected children shared the same maternal haplotype of their $\operatorname{del}(21 \mathrm{q})$, but a crossover was detected in the paternally inherited normal chromosome 21.
\end{abstract}

K. Wakui · Y. Fukushima $(\bowtie)$

Department of Medical Geneties, Shinshu University School of

Medicine, 3-1-1 Asahi, Matsumoto 390-8621, Japan

Tel. +81-263-37-2618; Fax +81-263-37-2619

e-mail: yfukush@sch.md.shinshu-u.ac.jp

K. Wakui · Y. Fukushima

Division of Clinical and Molecular Genetics, Shinshu University Hospital, Matsumoto, Japan

A. Toyoda $\cdot$ Y. Sakaki $\cdot$ M. Hattori

Genomic Sciences Center, RIKEN Yokohama Institute, Yokohama, Japan

T. Kubota

Department of Mental Retardation and Birth Defect Research,

National Institute of Neuroscience, National Center of Neurology

and Psychiatry, Tokyo, Japan

E. Hidaka $\cdot$ M. Ishikawa $\cdot$ T. Katsuyama

Department of Laboratory Medicine, Shinshu University Hospital, Matsumoto, Japan

Y. Fukushima

CREST, Japan Science and Technology Corporation, Kawaguchi, Japan
These data suggest that unmasking of deleterious genes on the paternally derived chromosome 21 of the two children as a result of the deletion may affect the extent of their mental retardation and/or sensorineural hearing loss. Usher syndrome $1 \mathrm{E}$ may be a candidate disease locus related to the sensorineural hearing loss of the first child.

Key words Chromosome $21 \cdot$ Partial monosomy chromosome $21 \cdot 21 \mathrm{q} 21 \cdot$ Fluorescence in situ hybridization $\cdot$ Mental retardation - Sensorineural hearing loss $\cdot$ Usher syndrome $1 \mathrm{E} \cdot$ USH1E

\section{Introduction}

Pure proximal monosomy for $21 \mathrm{q}$ is very rarely observed in humans, but a few cases have been reported to date (Wulfsberg et al. 1983; Reynolds et al. 1985; Roland et al. 1990; Korenberg et al. 1991; Huret et al. 1995; Ahlbom et al. 1996). Mild to moderate mental retardation is a common symptom in almost all the reported monosomy 21q21 cases, but other clinical features were not consistent. Korenberg et al. (1991) reported a boy with a de novo 21q11-q21.3 deletion, normal intelligence, minor anomalies, and epileptic seizures. There have also been reports of individuals with de novo or familial deletions involving similar regions of chromosome 21 , and with mild mental retardation and minor phenotypic abnormalities (Roland et al. 1990; Ahlbom et al. 1996). However, none of these cases were affected with sensorineural hearing loss.

Here we describe a familial 21q11.2-q21.3 deletion with variable phenotypic expression, including a phenotypically normal mother, one child with sensorineural hearing loss and moderate mental retardation, and another child with only mild mental retardation. We also report the results of breakpoint analysis of their chromosomes 21 by fluorescence in situ hybridization (FISH) using subregion-specific DNA clones on 21q and by haplotyping with polymorphic 21q markers. We discuss the variable phenotypes of these three individuals. 
Fig. 1. a Pedigree of the family, and $\mathbf{b}$ G-banded chromosomes 21 of the mother. The mother (I-2), and her two children (II-1 and II4) carry a $21 q$ deletion. Squares indicate male sex, circles indicate female sex; $y$, years; $m$, months. Asterisks indicate deleted chromosomes 21

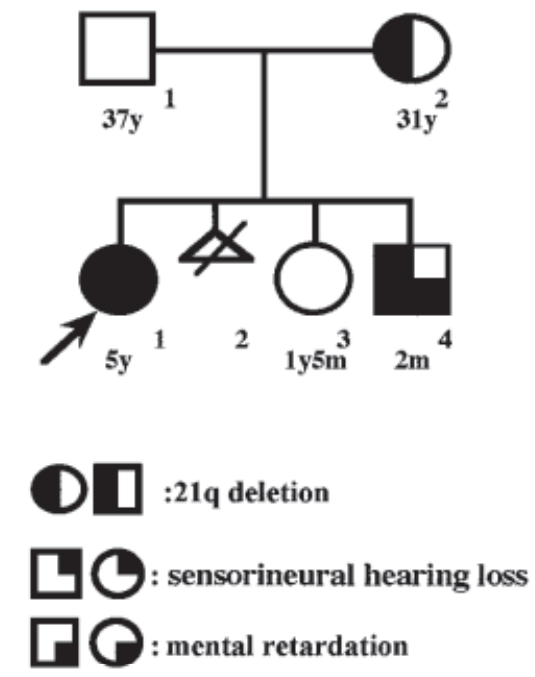

a

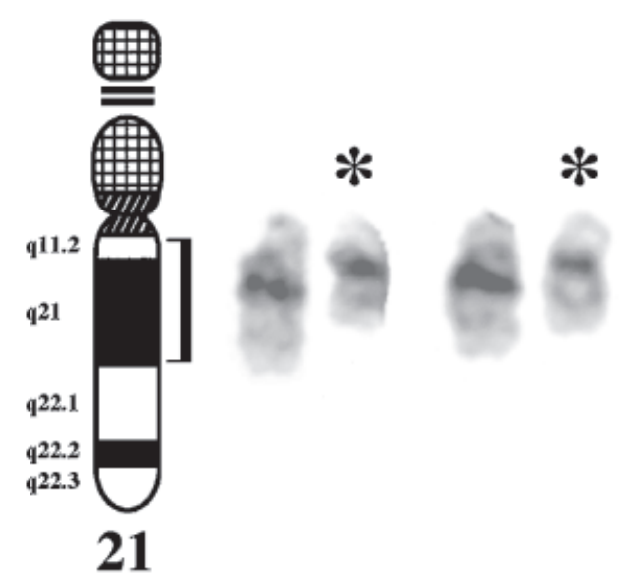

b

\section{Subjects and methods}

Clinical history

The proband was a 4-year-old girl (Fig. 1a, II-1) who presented with sensorineural hearing loss $(100 \mathrm{~dB})$. She was the first child of the phenotypically normal, healthy, and unrelated parents. The father (Fig. 1a, I-1) was 32 years old and the mother (Fig. 1a, I-2) 26 years old when the proband was born. Family history was negative for hearing impairment or mental retardation. The mother had contracted rubella at around 18 weeks of pregnancy; therefore, the girl's hearing impairment was initially attributed to the fetal rubella syndrome. This cause is, however, unlikely because the mother had rubella after the 17 th week of pregnancy. The girl, in addition, had strabismus and mild mental retardation. G-banded chromosomes of the girl showed a deletion of a 21q11.2-q21.3 segment. Chromosomes 21 of her father (Fig. 1a, I-1) were normal, but the mother (Fig. 1a, I-2) had a $21 \mathrm{q}$ deletion similar to that in the girl. The mother graduated from senior high school and worked as a store clerk before marriage. The maternal grandparents were not karyotyped. Because the mother had a varicella infection during the succeeding pregnancy, the second pregnancy was terminated (Fig. 1a, II-2). The subsequent pregnancy resulted in a liveborn girl (Fig. 1a, II-3), who was clinically checked by us when she was 12 months old. The girl was healthy, developing normally, appeared phenotypically normal, and had normal chromosomes.

The fourth pregnancy, monitored with amniocentesis at 16 weeks, showed a deleted chromosome 21 . At 19 weeks of pregnancy, the parents were referred to the genetics clinic at our university hospital. Ultrasonography of the fetus showed normal size without detectable abnormalities. We reexamined the mother's chromosomes by G-banding, Q-banding, and whole chromosome painting FISH. After receiving genetic counseling, including the provision of in- formation in the literature, the couple elected to continue the pregnancy. A boy was born at 39 weeks' gestation, weighing $2900 \mathrm{~g}$. The boy was examined at the age of 4 months; he had no malformations or hearing loss, but showed mild developmental delay (Fig. 1a, II-4).

\section{Cytogenetic analysis}

G- and Q-banding were carried out on peripheral blood lymphocyte chromosomes of this family. FISH analysis was performed using a whole chromosome painting probe for chromosome 21 (WCP21 SpectrumOrange-labeled, Vysis, Downers Grove, IL, USA) on the metaphase spreads from the mother. Separate images were collected for the $4^{\prime}-6^{\prime}$ diamidino-2-phenylindole counterstain of the chromosomes and merged with the hybridization signals using an image analysis system.

\section{FISH analysis using subregion-specific DNA clones}

FISH analyses of the chromosomes from the mother, first daughter, and first son, all with deleted 21q, were performed using subregion-specific DNA clones to determine their precise regions of deletion. We selected DNA clones mapped to 21q11-q22.2 as FISH probes by referring to the sequence information of chromosome 21 (Hattori et al. 2000). Ten clones (R15D13, R213O17, R114D22, R195K7, R195O17, R692A24, R92O22, R125G10, R45O19, and R666H8), which had not been described in the report of Hattori et al. (2000), were used as probes. These clones were screened from an RPCI-11 bacterial artificial chromosome library (Osoegawa et al. 2001) and closely mapped to distal borders of the deletion by alignment of their end sequences compared with the chromosome 21 sequence (Fig. 2). Two-color FISH analyses were performed with two of these clones as described previously (Wakui et al. 1999). 
Fig. 2. a Clones used as fluorescence in situ hybridization (FISH) probes and b FISH analysis in the mother. Clones in bold-faced type are those having confirmed a deletion in this family. Signal for R746N6 (arrowheads) appears only on the normal chromosome 21, and signal for R282I5 (arrows) appears on both the normal and deleted chromosomes 21

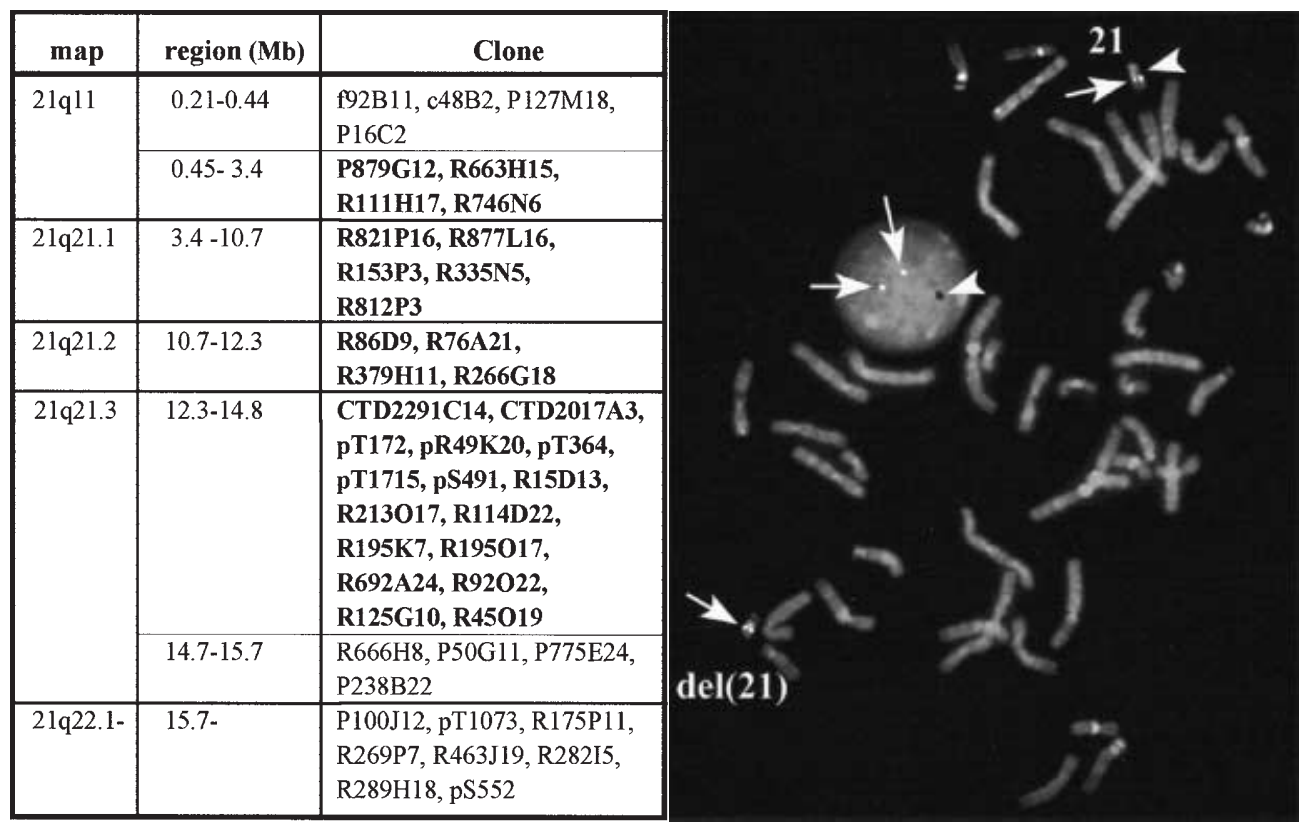

a

In brief, a probe was labeled with digoxigenin-11 deoxyuridine 5-triphosphate (dUTP) by nick translation and detected by anti-digoxigenin-rhodamine, Feb fragments, and the other probe was labeled with biotin-16dUTP and detected with avidin fluorescein isothiocyanate, respectively.

\section{Haplotype analysis}

Allelotypes of the parents and their three children were determined with a DNA-sequencer-assisted method, using 21 highly polymorphic microsatellite DNA markers at the proximal 21q region. Genomic DNA extracted from their peripheral blood was amplified by Polymerase Chain Reaction (PCR) using Cy5-labeled primers (Pharmacia, Stockholm, Sweden) under the following conditions: 35 cycles at $94^{\circ} \mathrm{C}$ for $30 \mathrm{~s}, 55^{\circ}-60^{\circ} \mathrm{C}$ for $30 \mathrm{~s}$, and $72^{\circ} \mathrm{C}$ for $30 \mathrm{~s}$, followed by a final extension at $72^{\circ} \mathrm{C}$ for $10 \mathrm{~min}$ in a thermocycler (model 9700, Perkin-Elmer, Shelton, CT, USA). PCR products were mixed with two kinds of suitable size markers (Cy5 sizer, Pharmacia) as an internal control and with loading dye. The mixed products were electrophoresed in $8 \%$ acrylamide gel $(7 \mathrm{M}$ urea and $1 \times$ Trisborate + ethylenediaminetetra acetate) and analyzed on an automated DNA sequencer (ALFexpress DNA sequencer, Pharmacia).

\section{Results}

Cytogenetic analysis

G- and Q-banding showed that the mother, the first daughter, and the son had the same deletion of 21q11.2-q21.3
[46,XX or XY,del(21)(q11.2q21.3)] (Fig. 1b). The WCP21 FISH signals were detected on normal and abnormal chromosomes 21 of the mother, but were not observed on any other chromosomes (data not shown).

FISH analysis using subregion-specific DNA clones

Deleted chromosome 21 in the two children and their mother was examined to define its breakpoints and extent using 45 subregion-specific DNA clones as FISH probes (Fig. 2). Clones (f92B11, c48B2, P127M18, and P16C2) that cover the $21 \mathrm{q} 11$ region $0.21-0.44 \mathrm{Mb}$ distal to the centromere (Fig. 2) hybridized to both the normal and deleted chromosomes 21 in the three individuals, indicating that this region remained in the deleted chromosome 21. All 29 clones covering $0.45-14.8-\mathrm{Mb}$ regions from the centromere (Fig. 2) hybridized to the normal but not to the deleted chromosome 21 in the three individuals. FISH signals for R666H8 again appeared in both the normal and aberrant chromosomes 21 in the three persons. These data indicated that the deletion in the family extends between positions $0.45 \mathrm{Mb}$ and $14.8 \mathrm{Mb}$ distal to the centromere of chromosome 21 .

\section{Haplotype analysis}

The results of haplotyping of 21 polymorphic marker loci in the five family members are shown in Fig. 3. In the mother, her first daughter, and her son, homozygous or hemizygous patterns were observed in a segment from the D21S215 locus $(0.46 \mathrm{Mb}$ from the centromere) to the D21S1442 locus $(14.4 \mathrm{Mb}$ from the centromere). Among these marker loci, D21S215, D21S1433, D21S11, CHLC.GATA22H11, 


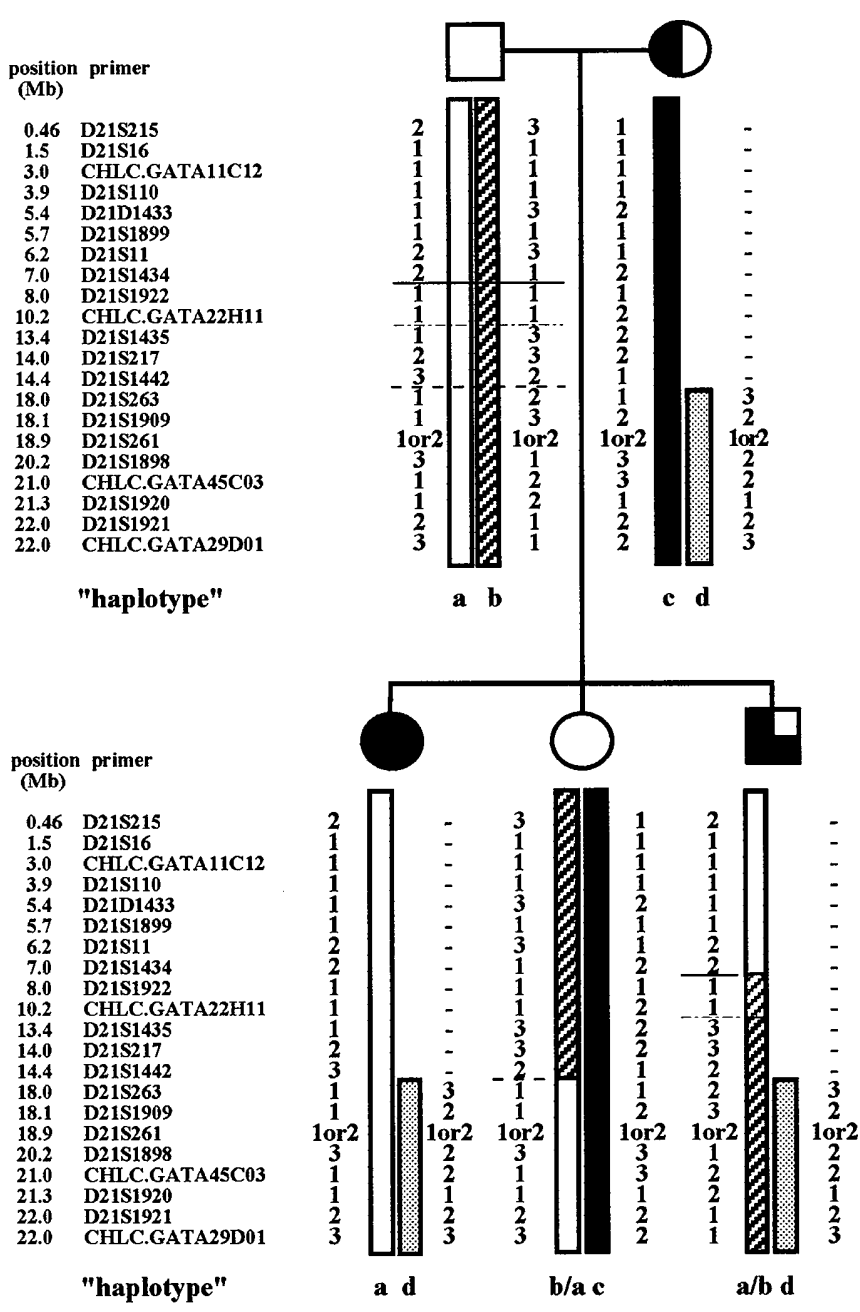

Fig. 3. Haplotype analysis of the family using 21 polymorphic markers at 21q11-q22. Open and hatched columns show each paternal haplotype set, filled columns show that of the maternal normal chromosome 21 , and shaded columns show that of the maternal deleted chromosome 21. Deletion from the D21S215 to the D21S1442 loci in the mother is deduced both from FISH data on her chromosomes and the extent of deletion in her affected children

D21S1435, and D21S1442 definitely showed paternal monoallelic patterns in the first daughter, and those plus the D21S217 locus demonstrated such monoallelic patterns in the son. On the other hand, both maternal and paternal alleles commonly present in the two children were at the D21S263, D21S1909, D21S261, D21S1898, and CHLC.GATA45C03 loci. Five other markers, D21S16, CHLC.GATA11C12, D21S110, D21S1899, and D21S1922, were uninformative because they showed homozygous patterns in all five family members.

\section{Discussion}

We herein reported a familial 14-Mb chromosomal deletion within the 21q11.2-q21.3 region, i.e., almost a half of the long arm of chromosome 21 . The deletion was transmitted from a phenotypically normal mother to her two children who had mental retardation and/or sensorineural hearing loss (Fig. 1). FISH analysis using subregion-specific DNA clones and haplotyping using microsatellite markers revealed that the extent of the deletion was identical in the three individuals, spanning between $0.33-0.46 \mathrm{Mb}$ and $14.6-$ $14.9 \mathrm{Mb}$ from the centromere (Fig. 2 and 3). There are two duplicated regions, each being $200 \mathrm{~kb}$ long (Potier et al. 1998), showing $96 \%$ identity to each other and located at the $188-377-\mathrm{kb}$ region of $21 \mathrm{q} 11.2$ and at the $14,795-$ 15,022-kb region of 21q22 (Hattori et al. 2000). Because the two 200-kb duplicated regions largely overlap the proximal and the distal breakpoints of the deletion observed in this family, respectively (Fig. 4), it is likely that the deletion in the family is causally related to the duplication. Both the elder daughter and the son in the family shared the identical paternal alleles from the D21S215 to the D21S1434 or CHLC.GATA22H11 loci, but the paternal chromosome 21 in the boy had apparently undergone a crossing over at a point between the D21S1434 and D21S1435 loci. A segment distal to the crossing-over site was derived from the paternal second chromosome 21 (Fig. 3). Single-nucleotide polymorphism analysis of this family supported this conclusion (data not shown).

Sensorineural hearing loss, which the first daughter of this family had, has not been indicated by any previous reports of pure proximal 21q deletion (Wulfsberg et al. 1983; Roland et al. 1990; Korenberg et al. 1991; Reynolds et al. 1985; Huret et al. 1995; Ahlbom et al. 1996). The two children of this family were affected with mental retardation and/or sensorineural hearing loss, and their mother was phenotypically normal, although the extent of their deletions was identical. Thus, the abnormal phenotypes in the children may represent unmasking of a mutated gene on the nondeleted, paternally derived chromosome 21 . The results of our haplotype analysis suggest that a recessive gene, probably related to mental retardation, exists between the 0.33 - and the $0.46-\mathrm{Mb}$ region around the proximal breakpoint and the 13.4-Mb region (D21S1435), because the two affected children shared the same paternal alleles between the two regions. Our data also suggest that another putative recessive gene responsible for hearing impairment exists between the 7.0-Mb region (D21S1434) and the 14.6to $14.9-\mathrm{Mb}$ region around the distal breakpoint, because the two children had different paternal alleles in this segment (Figs. 3 and 4).

No genes have been annotated in the two 200-kb duplicated regions and only 17 genes are known to be located in a segment corresponding to an approximate $14-\mathrm{Mb}$ deletion in this family (Hattori et al. 2002). Some of these genes may causally be related to mental retardation, because almost all patients with a $21 \mathrm{q}$ deletion reported so far have some degree of mental retardation.

The Usher syndrome type 1E (MIM *602097) locus (USH1E) was assigned between D21S1905 (the 6.6-Mb region) and D21S1913 (the 18.0-Mb region) loci at 21q21-q22, by homozygosity mapping in a consanguineous Moroccan family by Chaib et al. (1997). Usher syndrome is a group of clinically variable and genetically heterogeneous autosomal 
Fig. 4. A simplified $21 \mathrm{q} 11-\mathrm{q} 22$ genetic map with intervals studied. Clones selected for FISH analyses in this study are placed on the left. White letters on black rectangles are clones that were deleted in the family members with the $21 \mathrm{q}$ deletion. Proximal and distal breakpoints on 21q of this family are flanked by the highly conserved tow large duplicated regions

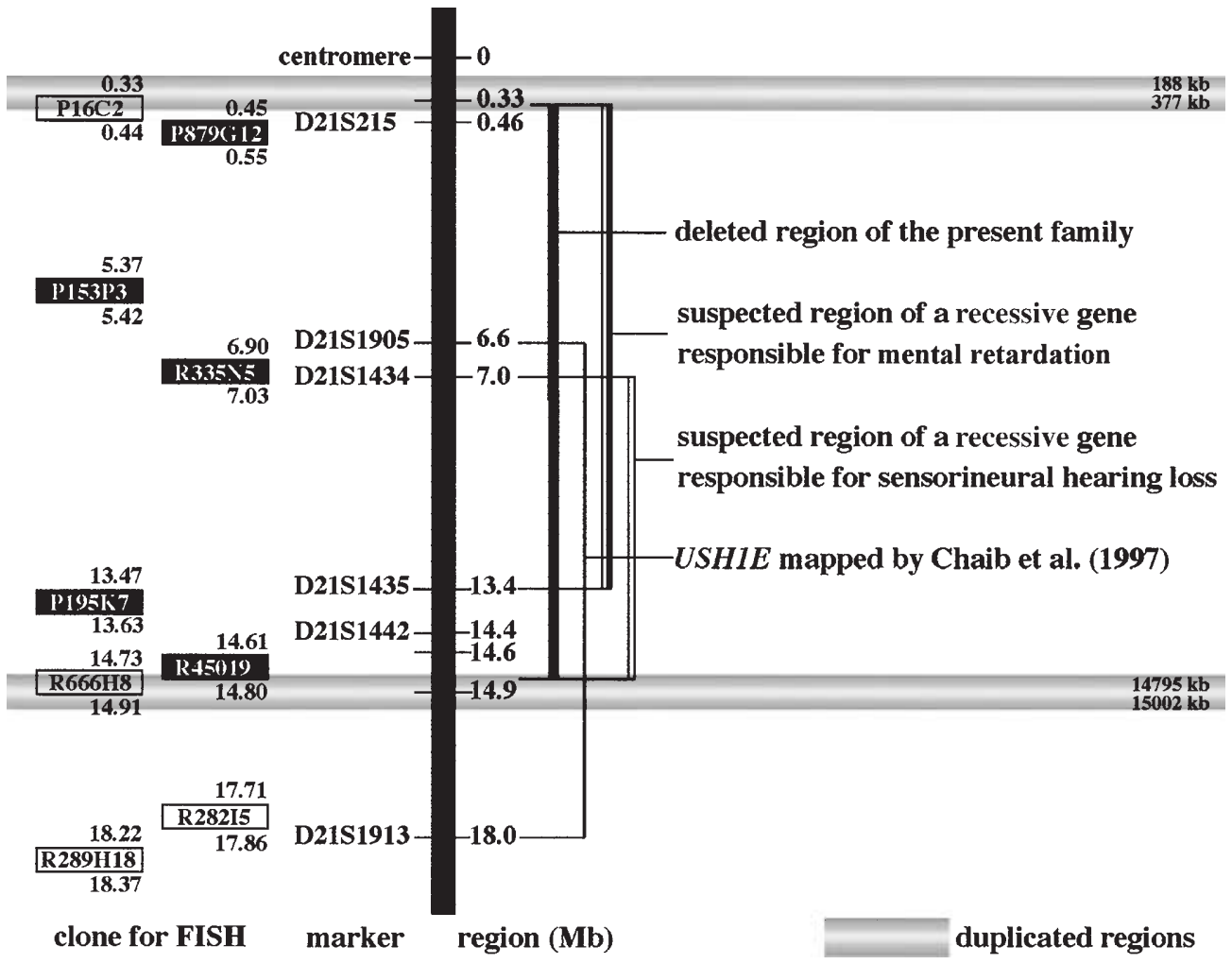

recessive disorders, and is characterized by congenital sensorineural hearing loss and pigmentary retinopathy. Retinopathy usually occurs in late childhood or adolescence and may lead to total blindness (Keats and Corey 1999). Regarding the hearing impairment of the first daughter, USH1E may be the candidate disease locus related to her sensorineural hearing loss, although such visual impairments have not yet been observed in her. Periodic ophthalmological checkups for retinopathies will be necessary for the first daughter.

It should be noted that the mother is entirely normal mentally and physically, even though a significantly large deletion was identified in one of her chromosomes 21 . The proximal half of $21 \mathrm{q}$ contains only 58 genes and represents a very gene-poor and less functional region (Hattori et al. 2000). This suggests that trisomies and partial monosomies involving this region are relatively well tolerated in humans, compared with those in other chromosomes.

Acknowledgments We are grateful to Dr. Misao Ohki and Dr. Hiroki Gomyo at National Cancer Center Research Institute for their kind provision of polymorphic markers and information, and to Dr. Tomoshi Okada and Dr. Kohji Horie at Nagano Red Cross Hospital for providing clinical information about the patients. We also thank Dr. Lisa G. Shaffer at Baylor College of Medicine; Dr. Todd D. Taylor at Genomic Sciences Center, RIKEN; Dr. Yu-ichi Goto at National Institute of Neuroscience, NCNP; and Professor Eric Johnson at Jichi Medical School for their helpful advice. This work was supported in part by grants from the Ministry of Education, Culture, Sports, Science and Technology, and from the Ministry of Health, Labor and Welfare, Japan.

\section{References}

Ahlbom BE, Sidenvall R, Anneren G (1996) Deletion of chromosome 21 in a girl with congenital hypothyroidism and mild mental retardation. Am J Med Genet 64:501-505

Chaib H, Kaplan J, Gerber S, Vincent C, Ayadi H, Slim R, Munnich A, Weissenbach J, Petit C (1997) A newly identified locus for Usher syndrome type I, USH1E, maps to chromosome 21q21. Hum Mol Genet 6:27-31

Hattori M, Fujiyama A, Taylor TD, Watanabe H, Yada T, Park HS, Toyoda A, Ishii K, Totoki Y, Choi DK, Groner Y, Soeda E, Ohki M, Takagi T, Sakaki Y, Taudien S, Blechschmidt K, Polley A, Menzel U, Delabar J, Kumpf K, Lehmann R, Patterson D, Reichwald K, Rump A, Schillhabel M, Schudy A, Zimmermann W, Rosenthal A, Kudoh J, Schibuya K, Kawasaki K, Asakawa S, Shintani A, Sasaki T, Nagamine K, Mitsuyama S, Antonarakis SE, Minoshima S, Shimizu N, Nordsiek G, Hornischer K, Brant P, Scharfe M, Schon O, Desario A, Reichelt J, Kauer G, Blocker H, Ramser J, Beck A, Klages S, Hennig S, Riesselmann L, Dagand E, Haaf T, Wehrmeyer S, Borzym K, Gardiner K, Nizetic D, Francis F, Lehrach H, Reinhardt R, Yaspo ML (2000) The DNA sequence of human chromosome 21. Nature 405:311-319

Huret JL, Leonard C, Chery M, Philippe C, Schafei-Benaissa E, Lefaure G, Labrune B, Gilgenkrantz S (1995) Monosomy 21q: two cases of del(21q) and review of the literature. Clin Genet 48:140-147

Keats BJ, Corey DP (1999) The usher syndromes. Am J Med Genet 89:158-166

Korenberg JR, Kalousek DK, Anneren G, Pulst SM, Hall JG, Epstein CJ, Cox DR (1991) Deletion of chromosome 21 and normal intelligence: molecular definition of the lesion. Hum Genet 87:112-118

Osoegawa K, Mammoser AG, Wu C, Frengen E, Zeng C, Catanese JJ, de Jong PJ (2001) A bacterial artificial chromosome library for sequencing the complete human genome. Genome Res 11:483-496

Potier M, Dutriaux A, Orti R, Groet J, Gibelin N, Karadima G, Lutfalla G, Lynn A, Van Broeckhoven C, Chakravarti A, Petersen M, Nizetic D, Delabar J, Rossier J (1998) Two sequence-ready contigs spanning the two copies of a $200-\mathrm{kb}$ duplication on human 21q: partial sequence and polymorphisms. Genomics 51:417-426 
Reynolds JF, Wyandt HE, Kelly TE (1985) De novo 21q interstitial deletion in a retarded boy with ulno-fibular dysostosis. Am J Med Genet 20:173-180

Roland B, Cox DM, Hoar DI, Fowlow SB, Robertson AS (1990) A familial interstitial deletion of the long arm of chromosome 21. Clin Genet 37:423-428

Wakui K, Tanemura M, Suzumori K, Hidaka E, Ishikawa M, Kubota T, Fukushima Y (1999) Clinical applications of two-color telomeric
FISH for prenatal diagnosis: identification of chromosomal translocation in five families with recurrent miscarriages or a child of multiple congenital anomalies. J Hum Genet 44:85-90

Wulfsberg EA, Carrel RE, Klisak IJ, O’Brien TJ, Sykes JA, Sparkes RS (1983) Normal superoxide dismutase-1 (SOD-1) activity with deletion of chromosome band 21q21 supports localization of SOD-1 locus to 21q22. Hum Genet 64:271-272 